\title{
Nonlinear oscillations of a viscoelastic cylindrical panel with concentrated masses
}

\author{
Dadakhan Khodzhaev D.A. ${ }^{1,}$, Rustamkhan Abdikarimov², and Nikolay Vatin ${ }^{3}$ \\ ${ }^{1}$ Tashkent Institute of Irrigation and Agricultural Mechanization Engineers, Tashkent, Uzbekistan \\ ${ }^{2}$ Tashkent Financial Institute, Tashkent, Uzbekistan \\ ${ }^{3}$ Peter the Great St. Petersburg Polytechnic University, Polytechnicheskaya st. 29, St. Petersburg, \\ 195251, Russian Federation
}

\begin{abstract}
Absract. The problems of oscillations of a viscoelastic cylindrical panel with concentrated masses are investigated, based on the Kirchhoff-Love hypothesis in the geometrically nonlinear statement. The effect of the action of concentrated masses is introduced into the equation of motion of the cylindrical panel using the $\delta$ function. To solve integro-differential equations of nonlinear problems of the dynamics of viscoelastic systems, a numerical method is suggested. With the Bubnov-Galerkin method, based on a polynomial approximation of the deflection, in combination with the suggested numerical method, the problems of nonlinear oscillation of a viscoelastic cylindrical panel with concentrated masses were solved. Bubnov-Galerkin's convergence was studied in all problems. The influence of the viscoelastic properties of the material and concentrated masses on the process of oscillations of a cylindrical panel is shown.
\end{abstract}

\section{Introduction}

With intensive progress in modern industry one of the most important problems in machine-building and engineering is the decrease in specific consumption of materials of the structures and machines. Saving in materials brings the necessity to produce a thinwalled structures. However the thinner the element the more flexible it is, and the more pronounced is its tendency to the buckling and the loss in stability. The latter is accompanied by the catastrophic growth of deformation and, as the rule, by the failure of structures. From this point of view, when manufacturing a light, strength and reliable structures, the most acceptable are the composite materials, which allow to improve considerably an operational characteristics of these structures and, sometimes, to manufacture the structures, which could not be realized with traditional materials. The procedure of the design and projecting of structures made of composite materials is rather complicated; it requires the account of their real properties. So, the development of effective algorithms of the solution of nonlinear problems of dynamic stability of shells, panels and plates made of composite materials presents the most actual problem.

\footnotetext{
*Corresponding author: dhodjaev@mail.ru
} 
Plates, panels and shells made of composite materials with fixed objects in the form of additional mass are widely used as a result of their high viscoelastic and strength characteristics. Therefore, while projecting the elements of the structure made of composite materials, it is very important to predict their dynamic characteristics which vary with their configuration, mass distribution, viscoelastic properties, etc.

In most cases, the role of additional mass is played by longitudinal and transverse ribs, straps, attachments, joints of devices and machines [1-3]. When theoretically considering problems of this type, it is convenient to interpret attached elements as additional mass, rigidly jointed to the system and concentrated in points. There are a number of publications devoted to the study of the oscillation and dynamic stability of elastic systems with concentrated masses $[3,4]$. In these works, the problems were solved in the linear state, and only a number of material properties of the structure were taken into account. Papers studying the problems of nonlinear oscillation and dyndmic stability of elastic cylindrical panels, carrying concentrated masses, are scarce [5]. Similar problems of oscillation and dynamic stability of an elastic and viscoelastic plates, cylindrical panels and shells in different state without concentrated masses were considered in [6-12].

It is well known, that the majority of composite materials possess pronounced viscoelastic properties [13-15]. Wide industrial application of new materials with viscoelastic properties and analysis of their dynamic behavior indicate an important effect of heterogenieties (of the type of concentrated mass) on their strength.

In spite of the studies carried out in this field, the peculiarities of the behavior of heterogeneous (in inertial relation), viscoelastic systems have not been thoroughly investigated. In these works, the problems were considered either with Voight's differential model $[16,17]$ or with Boltzmann-Volterra's integral model, where exponential kernels were taken as kernels of relaxation, although they could not describe the real processes occurring in shells, panels and plates at zero time.

One of the characteristic properties of this problem is the fact that after the use of Bubnov-Galerkin's method, the problem (both in linear and nonlinear states) is reduced to the solution of non-disintegrated systems of integro-differential equations with singular kernels, whose study may lead to additional complications. Thanks to a numerical method [18], based on the use of quadrature formula, it became possible to solve these systems. The method described provides sufficiently high accuracy of results obtained; it is universal and opens the possibility to solve a wide range of dynamic problems of the theory of viscoelasticity. It also economical from the point of view of computer time [19]. On the basis of this method, a great number of numeric results [20-23] were obtained, agreeing well with theoretical and experimental data [24].

The aim of this works is to study nonlinear problems of oscillation of viscoelastic cylindrical panel with concentrated masses.

\section{Methods}

Consider a gentle-sloping circular viscoelastic cylindrical panel with sides $a$ and $b$, of constant thickness $h$ and the radius of the median surface $R$, made of inhomogeneous orthotropic material, carrying a concentrated mass $M_{p}$, located at points $\left(x_{p}, y_{p}\right), p=1,2, \ldots$, I.

The equation of motion of the element of a viscoelastic cylindrical panel with concentrated masses has the following form

$$
B_{11}\left(1-R_{11}^{*}\right) \frac{\partial \varepsilon_{x}}{\partial x}+B_{12}\left(1-R_{12}^{*}\right) \frac{\partial \varepsilon_{y}}{\partial x}+2 B\left(1-R^{*}\right) \frac{\partial \gamma_{x y}}{\partial y}-
$$




$$
\begin{gathered}
-\left[\rho+\frac{1}{h} \sum_{i=1}^{I} M_{i} \delta\left(x-x_{i}\right) \delta\left(y-y_{i}\right)\right] \frac{\partial^{2} u}{\partial t^{2}}=0 \\
B_{22}\left(1-R_{22}^{*}\right) \frac{\partial \varepsilon_{y}}{\partial y}+B_{21}\left(1-R_{21}^{*}\right) \frac{\partial \varepsilon_{x}}{\partial y}+2 B\left(1-R^{*}\right) \frac{\partial \gamma_{x y}}{\partial x}- \\
-\left[\rho+\frac{1}{h} \sum_{i=1}^{I} M_{i} \delta\left(x-x_{i}\right) \delta\left(y-y_{i}\right)\right] \frac{\partial^{2} v}{\partial t^{2}}=0 \\
\frac{h^{2}}{12}\left\{\begin{array}{c}
B_{11}\left(1-R_{11}^{*}\right) \frac{\partial^{4}\left(w-w_{0}\right)}{\partial x^{4}}+\left[8 B\left(1-R^{*}\right)+B_{12}\left(1-R_{12}^{*}\right)+B_{21}\left(1-R_{21}^{*}\right)\right] \frac{\partial^{4}\left(w-w_{0}\right)}{\partial x^{2} \partial y^{2}}+ \\
\left.+B_{22}\left(1-R_{22}^{*}\right) \frac{\partial^{4}\left(w-w_{0}\right)}{\partial y^{4}}\right\}-k_{x}\left[B_{11}\left(1-R_{11}^{*}\right) \varepsilon_{x}+B_{12}\left(1-R_{12}^{*}\right) \varepsilon_{y}\right]- \\
-k_{y}\left[B_{22}\left(1-R_{22}^{*}\right) \varepsilon_{y}+B_{21}\left(1-R_{21}^{*}\right) \varepsilon_{x}\right]- \\
-\frac{\partial}{\partial x}\left\{\frac{\partial w}{\partial x}\left[B_{11}\left(1-R_{11}^{*}\right) \varepsilon_{x}+B_{12}\left(1-R_{12}^{*}\right) \varepsilon_{y}\right]+2 B \frac{\partial w}{\partial y}\left(1-R^{*}\right) \gamma_{x y}\right\}- \\
\left.+\rho+\frac{1}{h} \sum_{i=1}^{I} M_{i} \delta\left(x-x_{i}\right) \delta\left(y-y_{i}\right)\right] \frac{\partial^{2} w}{\partial t^{2}}=0
\end{array}\right.
\end{gathered}
$$

\section{Results and Discussion}

The results of calculations for nonlinear oscillation of viscoelastic cylindrical panel are given below. Calculations were performed using different physical and geometrical parameters (fig.1, 2). Here, if not written otherwise, initial data were taken in the following form: $w_{0}=10^{-3}, A=0.05, \alpha=0.25, \beta=0.05, q=0, \lambda=1, \theta=6, \mu=0.32, M_{1}=0.1$.

Numeric convergence of Bubnov-Galerkin's method has been studied. While determining the deflection values, the first seven harmonics $(N=7, M=1)$ were retained. Results show that a further increase in the number of components does not greatly influence the amplitude of oscillations of the viscoelastic panel.

Figure 1 shows the relationship between the deflection and the time of the median point of elastic $(A=0$ - curve 1$)$ and viscoleastic panel $(A=0.05,0.1$ - curves 2,3$)$. As can be seen from the figure, the account of the viscoelastic properties of the material of the panel under free oscillations $(q=0)$ leads to damping of the oscillation process. Although the solution of elastic and viscoelastic problems at zero times does not differ from each other, as time unfolds, the viscoelastic properties have a more pronounced influence. The results of the study show that the increase in rheological parameter $A$ yields a decrease in the amplitude and frequency of oscillations. 


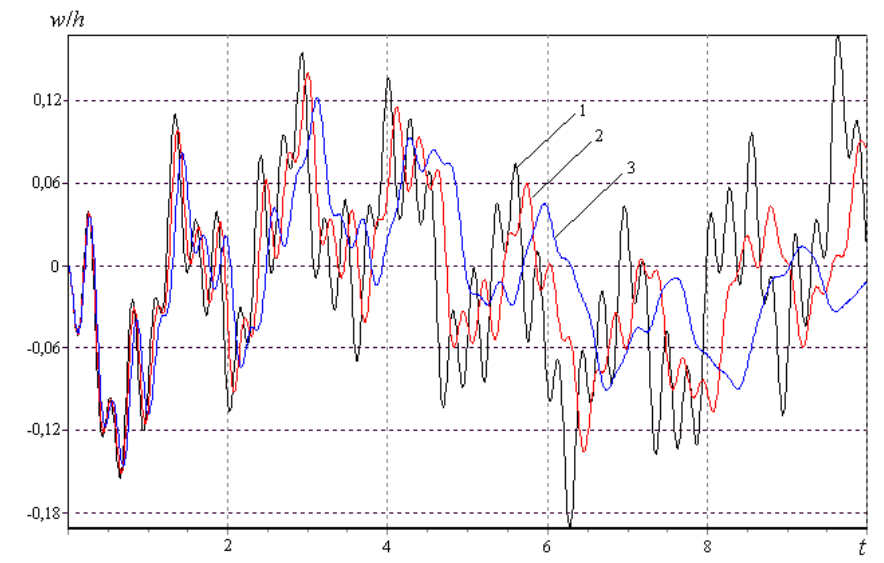

Fig.1.

Figure 2 presents the results of a study of the influence of a concentrated mass on the oscillation process of a viscoelastic panel. Here, curve 1 corresponds to the case without concentrated mass $\left(M_{1}=0\right)$; curves 2,3 and 4 when the mass is concentrated at the median point of the panel and its parameter equals $M_{1}=0.1,0.3,0.5$, respectively. As seen from the figure, an increase in parameters of concentrated mass yields a decrease in amplitude and frequency of oscillation. This is explained by the fact that, in a given case, a concentrated mass acts as a damper and an increase in mass yields more intensive damping of oscillations.

The influence of a geometrical nonlinearity on the frequency and amplitude of oscillations of a viscoelastic panel was studied too. Calculations show that for the case when the oscillations of a viscoelastic cylindrical panel are considered without the external loads and initial imperfections, the results obtained from the solution of linear and nonlinear problems will coincide. So in those cases the problems may be studied in a linear statement. However, with an increase in relationships between the geometrical parameters $\lambda$ of a panel with external static transverse loads $q$ and initial imperfections $w_{0}$, the differences are observed in the results obtained. In these cases the problems should be solved in nonlinear state.

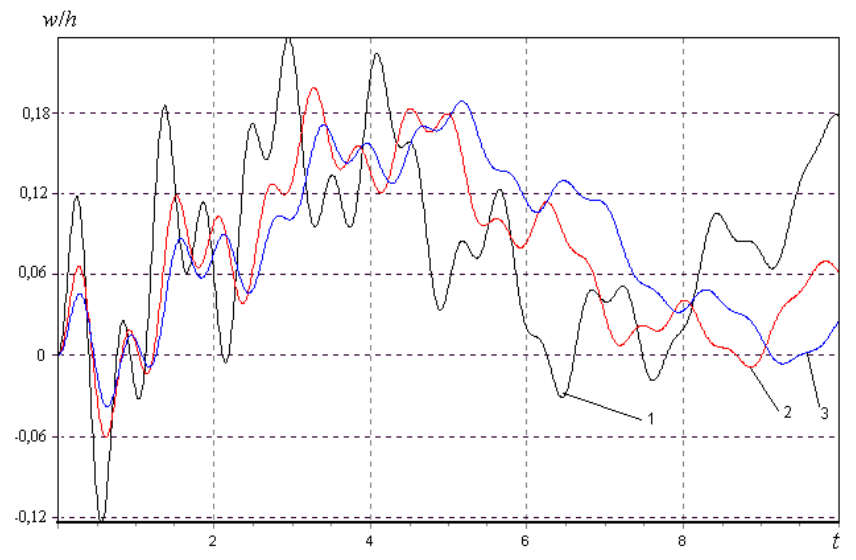

Fig.2. 


\section{Conclusions}

Investigation of nonlinear problems of oscillations of viscoelastic cylindrical panels with a concentrated mass shows that:

- an increase in concentrated mass leads to a sharper drop in the amplitude and frequency of oscillations than in elastic case;

- similar to the elastic case, the greater the distance of the concentrated mass from the center of the panel, the higher the frequency of oscillations;

- results obtained with exponential kernels coincide with the results of the elastic problem and could not be used as a kernel of relaxation, describing real viscoelastic properties of the material of the cylindrical panel;

- in design, with different geometrical and physical parameters of the cylindrical panel, it is necessary to choose the corresponding theory (linear and nonlinear theories).

\section{References}

1. I.V. Andrianov, V.A. Lesnichaya, L.I. Manevich, Averaging method in a statics and dynamics of ribbed shells, Nauka, Moscow (1985).

2. I.Ya. Amiro, and others, Oscillations of ribbed shells of rotation, Naukova Dumka, Kiev (1988).

3. L.V. Andreev, A.L. Dyshko, I.D. Pavlenko, Dynamics of plates and shells with the concentrated masses, Mashinostroenie, Moscow (1988).

4. C.L. Amba-Rao, Journal of Applied Mechanics, 31, 550-551, (1964).

5. P.A. Bondarev, Ukrainian mathematical journal, 1, 61-66 (1974).

6. Chang-jun Cheng, Neng-hui Zhang, Applied Mathematics and Mechanics, 22(1), 1-9 (2001).

7. Y.X. Sun, S.Y. Zhang, International Journal of Mechanical Sciences, 43, 1195-1208 (2001).

8. D.H. van Campen, V.P. Bouwman, G.Q. Zhang, J. Zhang, B.J.W. ter Weeme, International Journal of Non-linear Mechanics, 37, 659-667 (2002).

9. G. Cederbaum, D. Touati, International Journal of Non-linear Mechanics, 37, 757-762 (2002).

10. J. Awrejcewicz, V.A. Krys'ko, Nonclassical thermoelastic problems in nonlinear dynamics of shells, Applications of the Bubnov-Galerkin and finite difference numerical methods, Springer-Verlag (2003).

11. S.K. Sahu, P.K. Datta, Journal of Engineering mechanics, 129(11), 1245-1253 (2003).

12. L.R. Kumar, P.K. Datta, D.L. Prabhakara, Composite Structures, 60, 171-181 (2003).

13. A.A. Il'yushin, B.E. Pobedrya, Fundamentals of the mathematical theory of thermoviscoelasticity, Nauka, Moscow (1970).

14. M.A. Koltunov, Creep and relaxation, Visshaya shkola, Moscow (1976).

15. Yu.N. Rabotnov, Creep of elements of designs, Nauka, Moscow (1966).

16. T. Kocatürk, S. Sezer, C. Demir, Journal of Sound and Oscillation, 278, 789-806 (2004).

17. K. Cabanska-Placzkiewicz, Strength of materials, 34(2), 165-180 (2002).

18. F.B. Badalov, Kh. Eshmatov, M. Yusupov, Soviet Applied mathematics and mechanics, 51, 867-871 (1987). 
19. Kh. Eshmatov, Integrated method of mathematical modelling of problems of dynamics of viscoelastic systems, Avtoreferat diss. doc. teh. nauk, Kiev (1991).

20. A.F. Verlan, R.A. Abdikarimov, Kh. Eshmatov, Electronic modeling, 32 (2), 3-14 (2010).

21. R.A. Abdikarimov, V.M. Zhgutov, Magazine of Civil Engineering, 6, 38-47 (2010).

22. R.A. Abdikarimov, V.M. Zhgoutov, Magazine of Civil Engineering, 6, 12-22 (2011).

23. R.A. Abdikarimov, Kh. Eshmatov, Sh.P. Bobanazarov, D.A.Hodzhaev, B.Kh. Eshmatov, Magazine of Civil Engineering, 3, 59-70 (2011).

24. A.S. Volmir, The nonlinear dynamics of plates and shells, Nauka, Moscow (1972). 